\title{
Initial Experience of Laparoscopic Hepatectomy for Intrahepatic Duct (IHD) Stones: Comparison with Open Hepatectomy
}

\author{
Joon Sang Lee, M.D., Yoo Shin Choi, M.D., Ph.D., Seung Eun Lee, M.D., Ph.D. \\ Department of Surgery, Chung-Ang University College of Medicine, Seoul, Korea
}

Purpose: The aim of this study is to analyze our initial experiences with laparoscopic hepatectomy for treatment of intrahepatic duct (IHD) stones and to compare the outcomes of laparoscopic hepatectomy and open hepatectomy.

Methods: From March 2010 to December 2012, for treatment of IHD stones we performed laparoscopic hepatectomy in seven cases (LH group), which consisted of four cases of left lateral sectionectomy and three cases of left hepatectomy, and open hepatectomy was performed in nine cases $\mathrm{CH}$ group), which consisted of seven cases of left lateral sectionectomy and two cases of left hepatectomy. Because there was no case of right hepatectomy in the LH group, we excluded a case of right hepatectomy in the $\mathrm{OH}$ group. Retrospective analysis of the clinical outcomes was performed with exclusion of one case of right hepatectomy.
Results: There was no significant difference in male to female ratio, mean age, and mean operation time. The mean postoperative hospital stay of the LH group was significantly shorter than that of the $\mathrm{OH}$ group $(10.9 \pm 4.7$ vs. $22.0 \pm 9.0$ days, $p=0.007)$. There were two cases of intra-abdominal fluid collection in the $\mathrm{OH}$ group, but no case in the LH group. Remnant stones were detected in one patient of the LH group and two patients of the $\mathrm{OH}$ group. The initial success rate of stone clearance was $85.7 \%$ in the $\mathrm{LH}$ group and $77.8 \%$ in the $\mathrm{OH}$ group. During a mean follow-up period of 13 months (range, four to 25 months), recurrent stone was detected in one case of the $\mathrm{OH}$ group.

Conclusion: Laparoscopic surgery could be an effective treatment modality for management of IHD stones in select patients.

Key words: Bile ducts, Calculi, Laparoscopy, Hepatectomy

\section{INTRODUCTION}

Hepatolithiasis is a common disease in Southeast Asia and complete removal of stones and maintenance of adequate biliary flow are the bases of its treatment. ${ }^{1}$ Although there are various treatment modalities for this disease, hepatectomy seems to be the most effective treatment modality because it can remove the stones and the ductal strictures simultaneously in one operative procedure. ${ }^{2,3}$ With the refinement of laparoscopic instruments and accumulated experience in both laparoscopic surgery and hepatic surgery, an increasing number of reports on laparoscopic hepatectomy ( $\mathrm{LH})$ with favorable results have been documented. ${ }^{4}$ Until now, the LH for intrahepatic duct (IHD) stone has been rarely reported on, ${ }^{1-3}$ because there have been difficulties in LH for IHD stones because of adhesion of adjacent tissue or distorted anatomy resulting from recurrent inflammation. ${ }^{1}$ In this study, we analyzed our experiences with LH for hepatolithiasis to evaluate its feasibility.

Received September 1, 2014, Revised 1st, October 6, 2014; 2nd, October 9, 2014, Accepted October 10, 2014

※ Corresponding author : Seung Eun Lee

Department of Surgery, Chung-Ang University College of Medicine, 224-1 Heukseok-dong, Dongjak-gu, Seoul 156-755, Korea

Tel : +82-2-6299-3121, Fax : +82-2-824-7869

E-mail : selee508@ cau.ac.kr

\section{MATERIALS AND METHODS}

\section{1) Patients}

From March 2010 to December 2012, 17 patients with IHD stones were treated in our institute. Of the 17 patients, 7 patients underwent LH (LH group) and 10 patients underwent open hepatectomy ( $\mathrm{OH}$ group). The indications for performing LH were (1) stones associated with moderate to severe stricture or dilatation of the IHD, (2) IHD stones associated with parenchymal atrophic change, and (3) impacted stones in the unilateral hemiliver. LH group was consisted of 4 cases of left lateral sectionectomy and 3 cases of left hepatectomy, and $\mathrm{OH}$ group was consisted of 7 cases of left lateral sectionectomy, 2 cases of left hepatectomy. Because there was no case of right hepatectomy in LH group, we excluded right hepatectomy case of $\mathrm{OH}$ group in our study. Finally we compared 7 cases of LH group and 9 cases of $\mathrm{OH}$ group. These patients were followed up for $2 \sim 25$ months (mean=13 months).

\section{2) Operative technique}

LH was performed under general anesthesia with the patient in the supine position. The position was changed according to resection plane. A $11 \mathrm{~mm}$ trocar was inserted below the umbilicus and a $\mathrm{CO} 2$ pneumoperitoneum was established and 
maintained below $12 \mathrm{mmHg}$. A $30^{\circ}$ angle rigid or flexible scope was introduced. Under direct vision, $12 \mathrm{~mm}$ trocar was inserted below the left side of xiphoid process, two $5 \mathrm{~mm}$ trocars were inserted below the right, left costal margin (Fig. 1). The operator and cameraman stood on the right side of the patient and the first assistant on the left side. Parenchymal dissection of superficial portion was done by ultrasonic shears (Harmonic scalpel, Ethicon, Cincinnati, OH, USA) and deep portion was done by laparoscopic cavitron ultrasonic surgical aspirator (CUSA, Valleylab, Boulder, CO, USA). Glisson' pedicle was transsected by a vascular endo-GIA or hepatic artery and portal veins were ligated and divided via individual dissection. In the preoperative study, if the stones were presumed to be located close to the resection line, then the left hepatic duct was divided and intraoperative choledochoscopy was performed to confirm residual stones and extract them. Then the duct was closed with intracorporeal sutures. In the preoperative study, if the stones were located far from the expected resection line, the left hepatic duct duct was transected with an endo-GIA. Hepatic vein was divided by a vascular endo- GIA. Once the resected specimen was completely divided, it was inserted into a vinyl bag and then extracted through incision that was created by extending umbilical trocar site.

$\mathrm{OH}$ was performed via upper midline incision for left lateral sectionectomy and right subcostal incision with midline extension. After mobilization of the liver, parenchymal was transected using cavitron ultrasonic surgical aspirator (CUSA, Valleylab, Boulder, CO, USA). Hepatic artery and portal vein were ligated and divided via individual dissection. The left hep-

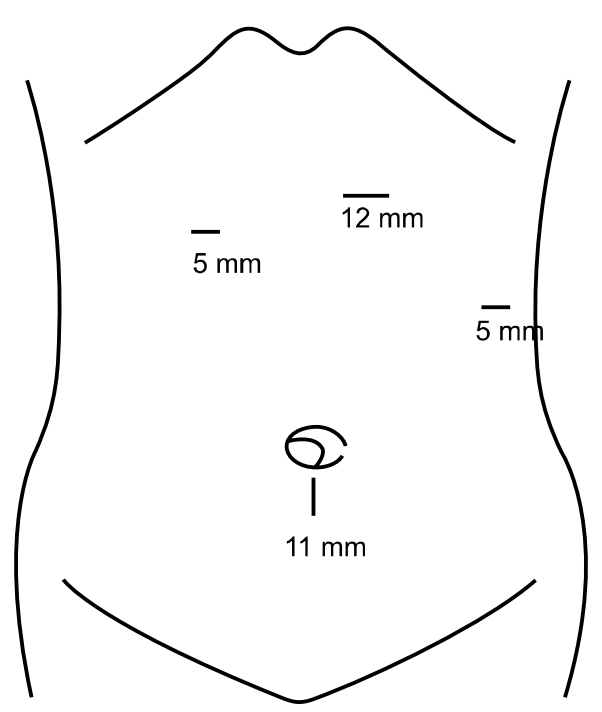

Fig. 1. Positioning of the trocars. The subumbilical port was extended for specimen extraction. atic vein was clamped and sutured with 5-0 prolene.

\section{3) Follow-up examination and assessment of stone clearance}

In all patients, abdominal CT scans and liver function tests were performed at postoperation 1 week, 3 month, 6 months, and 1 or 2 years at the outpatient clinic. Final stone clearance and the physical status of the patients were investigated by reviewing these follow-up examinations.

\section{4) Statistical analysis}

Continuous variables were compared using an independent sample, 2-tailed Student's t-test. Discrete variables were analyzed with the Chi square test. A $p$ value of less than 0.05 was considered significant.

\section{RESULTS}

\section{1) Patient characteristics}

Patient characteristics in this study are showed in Table 1. There was no significant difference in age, gender, BMI, and previous abdominal operation history between the two groups.

\section{2) Perioperative outcomes}

The mean operating time was similar between two groups (Table 2). No case was converted to the open procedure in $\mathrm{LH}$ group. Intraoperative transfusion ( 0 case vs.5 cases, $p=0.017$ ) and length of hospital stay (10.9 \pm 4.7 days vs. $22.0 \pm 9.0$ days, $p=0.007$ ) were significant shorter in LH group than $\mathrm{OH}$ group. There were no significant differences in operating time, intraoperative transfusion, and length of hospital stay whether the patient had previous abdominal operation history or not. There were 2 cases of intra-abdominal fluid collection in $\mathrm{OH}$ group, but no case in LH group. Remnant stones were detected in 1 patient of LH group and 2 patients of $\mathrm{OH}$ group. The initial success rate of stone clearance was $85.7 \%$ (6 of 7) in LH group

Table 1. Patient characteristics

\begin{tabular}{lccc}
\hline & LH $(\mathrm{n}=7)$ & OH $(\mathrm{n}=9)$ & $p$ value \\
\hline Age & $56.1 \pm 5.9$ & $59.8 \pm 9.0$ & 0.67 \\
Gender $(\mathrm{M}: \mathrm{F})$ & $2: 5$ & $2: 7$ & 0.68 \\
BMI $\left(\mathrm{kg} / \mathrm{m}^{2}\right)$ & $24.1 \pm 3.9$ & $23.2 \pm 3.3$ & 0.78 \\
Past history of abdominal & $4^{*}$ & $5^{\dagger}$ & 0.79 \\
$\quad$ surgery & & & \\
\hline
\end{tabular}

*3 cases of cholecystectomy, 1 case of cholecystectomy and CBD exploration; ${ }^{\dagger} 4$ case of cholecystectomy, 1 case of cholecystectomy and Roux-en-Y choledochojejunostomy. 
Table 2. Perioperative outcomes

\begin{tabular}{lccc}
\hline & LH $(\mathrm{n}=7)$ & $\mathrm{OH}(\mathrm{n}=9)$ & $p$ value \\
\hline Operation & & & 0.464 \\
Lt. lateral sectionectomy & 4 & 7 & \\
Lt. hepatectomy & 3 & 2 & \\
Operation time (min) & $212 \pm 66$ & $229 \pm 66$ & 0.619 \\
Conversion to open & 0 & & \\
Intraoperative transfusion & 0 & 5 & 0.017 \\
Hospital stay (days) & $10.9 \pm 4.7$ & $22.0 \pm 9.0$ & 0.007 \\
Complication (total) & 1 & 3 & 0.372 \\
Fluid collection & 0 & $2 *$ & \\
Wound problem & 1 & 1 & \\
Combined cholangiocarcinoma & 0 & 0 & 1.00 \\
Remnant stones (+) & 1 & 2 & 0.77 \\
Mortality & 0 & 0 & 1.00 \\
Follow up (months) & $12.1 \pm 9.2$ & $11.1 \pm 8.2$ & 0.815 \\
Recurrent stones & 0 & 1 & 0.301 \\
\hline
\end{tabular}

*PCD insertion; ${ }^{\dagger}$ Stone removal using choledocoscopy.

and $77.8 \%$ (7 of 9) in $\mathrm{OH}$ group. Remnant stones were removed successfully by percutaneous transhepatic cholangioscopy in LH group. During follow up period, recurrent stone was found in 1 case of $\mathrm{OH}$ group. Therefore, the final success rate of stone clearance was $100 \%$ (7 of 7) in LH group and $88.9 \%$ (8 of 9 ) in $\mathrm{OH}$ group.

\section{DISCUSSION}

Although there are various treatment modalities for IHD stones, ${ }^{1-3}$ it is difficult to treat because of the high rate of remnant or recurrent stones. Among various treatment modalities, hepatectomy seems to be the most advantageous procedure as it can remove IHD stones and ductal stricture, and resect hepatic parenchyma with irreversible changes, and removal of potential intrahepatic bile duct cancer, simultaneously. ${ }^{1-3}$ With the refinement of laparoscopic instruments and accumulated experience in laparoscopy, an increasing number of reports on laparoscopic liver resection for various hepatic lesions have been reported. ${ }^{4}$ However, there has been only a limited number of studies ${ }^{1-3}$ on LH for IHD stones. It might be because LH for IHD stones seems to be more technically demanding than LH for tumors. ${ }^{3}$ Severe perihepatic adhesion and the anatomic distortion resulting from the recurrent inflammation associated with IHD stones are frequently encountered during an operation. ${ }^{1,3}$ These may complicate the mobilization of the liver and the dissection, thus increasing the operation time and the intraoperative blood loss. However, if these limitations could be overcome, LH could be a good therapeutic option for those IHD stones because LH can offer the advantages of laparoscopic surgery, including less postoperative pain, early recovery and good cosmesis, as well as achieving the same therapeutic goal as the $\mathrm{OH}^{3}$ In this study, when compared with the results of the previously reported literature, ${ }^{1-3,5-8}$ our current data showed a similar outcome including stone clearance rate and postoperative morbidity. Furthermore, there was no significant difference in operation time between the LH group and $\mathrm{OH}$ group and intraoperative transfusion was significantly lower in the LH group. Although Pringle's maneuver was not used in our cases, the magnification through the endoscope might possibly allow a more precise surgical technique and the positive pressure of the abdomen might in turn minimised potential blood loss. Because our data has relatively short follow- up period of small number of patients, further long-term follow-up for more patients should be done to evaluate the stone recurrence rate after LH. Our data showed that LH could be performed safely and effectively for IHD stones with potential benefits of minimally invasive surgery such as shorter hospital stay and less intraoperative transfusion comparing with $\mathrm{OH}$. Furthermore, our current data showed a similar outcome for the stone clearance rate in LH group. Therefore, LH could be an effective treatment modality for the management of IHD stones in selected patients.

\section{REFERENCES}

1) Jeong $\mathrm{CY}$, Kim $\mathrm{KJ}$, Hong $\mathrm{SC}$, et al. Laparoscopic left hemihepatectomy for left intrahepatic duct stones. J Korean Surg Soc 2012;83(3):149-154.

2) Namgoong JM, Kim KH, Park GC, et al. Comparison of laparoscopic versus open left hemihepatectomy for left-sided hepatolithiasis. Int J Med Sci 2014;11(2):127-133.

3) Yoon YS, Han HS, Shin SH, Cho JY, Min SK, Lee HK. Laparoscopic treatment for intrahepatic duct stones in the era of laparoscopy: laparoscopic intrahepatic duct exploration and laparoscopic hepatectomy. Ann Surg 2009;249(2):286-291.

4) Simillis C, Constantinides VA, Tekkis PP, et al. Laparoscopic versus open hepatic resections for benign and malignant neoplasms--a meta-analysis. Surgery 2007;141:203-211.

5) Chen DW, Tung-Ping Poon R, Liu CL, Fan ST, Wong J. Immediate and long-term outcomes of hepatectomy for hepatolithiasis. Surgery 2004;135:386-393.

6) Lee SE, Jang JY, Lee JM, Kim SW. Selection of appropriate liver resection in left hepatolithiasis based on anatomic and clinical study. World J Surg 2008;32(3):413-418.

7) Lee TY, Chen YL, Chang HC, Chan CP, Kuo SJ. Outcomes of hepatectomy for hepatolithiasis. World J Surg 2007;31: 479-482.

8) Uchiyama K, Kawai M, Ueno M, Ozawa S, Tani M, Yamaue $\mathrm{H}$. Reducing residual and recurrent stones by hepatectomy for hepatolithiasis. J Gastrointest Surg 2007;11:626-630. 